OPEN ACCESS

Edited by:

Silvia C. Formenti,

Cornell University, United States

Reviewed by:

Michele Mondini,

Institut Gustave Roussy, France

Encouse Golden,

Cornell University, United States

*Correspondence:

Kwan-Hwa Chi khchi45@gmail.com

Specialty section:

This article was submitted to

Radiation Oncology,

a section of the journal

Frontiers in Oncology

Received: 07 August 2019

Accepted: 14 February 2020

Published: 10 March 2020

Citation:

Chi M-S, Mehta MP, Yang K-L, Lai $H-C$, Lin Y-C, Ko H-L, Wang Y-S, Liao K-W and Chi K-H (2020) Putative Abscopal Effect in Three Patients Treated by Combined Radiotherapy and Modulated Electrohyperthermia.

Front. Oncol. 10:254.

doi: $10.3389 /$ fonc. 2020.00254

\section{Putative Abscopal Effect in Three Patients Treated by Combined Radiotherapy and Modulated Electrohyperthermia}

\author{
Mau-Shin Chi ${ }^{1,2}$, Minesh P. Mehta ${ }^{3}$, Kai-Lin Yang ${ }^{1,4}$, Hung-Chih Lai ${ }^{5}$, Ying-Chu Lin ${ }^{5}$, \\ Hui-Ling Ko ${ }^{1}$, Yu-Shan Wang ${ }^{1,6}$, Kuang-Wen Liao ${ }^{6}$ and Kwan-Hwa Chi ${ }^{1,7 *}$ \\ ${ }^{1}$ Department of Radiation Therapy and Oncology, Shin Kong Wu Ho-Su Memorial Hospital, Taipei, Taiwan, ${ }^{2}$ Ph.D. Degree \\ Program of Biomedical Science and Engineering, National Chiao-Tung University, Hsinchu, Taiwan, ${ }^{3}$ Department of Radiation \\ Oncology, Miami Cancer Institute, Miami, FL, United States, ${ }^{4}$ School of Medicine, Fu Jen Catholic University, New Taipei City, \\ Taiwan, ${ }^{5}$ Department of Hematology and Oncology, Shin Kong Wu Ho-Su Memorial Hospital, Taipei, Taiwan, ${ }^{6}$ Institute of \\ Molecular Medicine and Bioengineering, National Chiao-Tung University, Hsinchu, Taiwan, ${ }^{7}$ School of Medicine, National \\ Yang-Ming University, Taipei, Taiwan
}

Purpose: True abscopal responses from radiation therapy are extremely rare; the combination of immune checkpoint inhibitors with radiation therapy has led to more reports of the abscopal effect, but even in this setting, the genuine magnitude remains unknown and is still considered generally uncommon. We report the occurrence of what appears to be putative, durable abscopal tumor responses with associated auto-immune systemic reactions resulting from the combination of local radiotherapy (RT) and modulated electrohyperthermia (mEHT).

Materials and Methods: Data from advanced cancer patients treated palliatively with RT and mEHT between January and December 2017 were collected as part of a post-marketing safety monitoring program of mEHT therapy. We specified a minimum RT dose of 30 Gy and at least four mEHT treatments for reporting toxicities, which was the primary aim of the larger study.

Results: Thirty-three patients treated with RT and mEHT, both applied to the same lesion, were included. The median RT dose was $45.5 \mathrm{~Gy}$ in 20 fractions (fxs) and the median number of mEHT treatments was 12 (range, 4-20). Most patients had subsequent systemic therapy after one course of RT and mEHT. Three patients $(9.1 \%)$ developed autoimmune toxicities. Case number 1 received RT and mEHT only; case number 2 had two cycles of concurrent low dose chemotherapy during RT; and case number 3 received concurrent immune checkpoint inhibitors. None of the three patients received any further systemic treatment due to obvious treatment-related autoimmune reactions which occurred rapidly after RT; one had autoimmune hepatitis, one had dermatitis herpetiformis and the third developed severe myasthenia gravis. Interestingly, what we surmise to be long-lasting abscopal responses outside the irradiated area, were noted in all three patients.

Conclusion: RT combined with mEHT could putatively result in enhancing immune responsiveness. These preliminary observational findings lead to the generation of a hypothesis that this combination induces both an in-situ, tumor-specific immune reaction 
and an anti-self-autoimmune reaction, in at least a small proportion of patients, and of those who experience the auto-immune response, tumor response is a concomitant finding. Mechanisms underlying this phenomenon need to be investigated further.

Keywords: modulated electrohyperthermia, immunotherapy, radiotherapy, abscopal effect, immune-related adverse events

\section{INTRODUCTION}

Local hyperthermia (HT) has long been regarded as an effective radio-sensitizer (1). Modulated electrohyperthermia (mEHT) therapy is one form of hyperthermia (2). mEHT utilizes the biophysical differences between malignant and normal cells for cancer-cell specific selective energy deposition, believed to be due to the lower impedance on the transmembrane protein clusters of malignant cells (3). The modulated electromagnetic frequency spectrum of $13.56 \mathrm{MHz}$ from mEHT is similar to the alternating electrical field generated from tumor treating field therapy (TTField, Novocure, Inc.) (4-6). mEHT applies lower power than conventional HT, thus interstitially measured average temperature is relatively low, around $39.5^{\circ} \mathrm{C}$. However, the corresponding transmembrane temperature differential across a cell is often quiet high (7). This transmembrane thermal stress destabilizes cell membranes, resulting in necrosis, and also enhanced apoptosis (8-11). This effect has been shown to enhance the release of heat shock proteins (HSPs), produce damage-associated molecular patterns (DAMP) and leads to increased immunogenicity, thereby mediating immunogenic cell-death (12). The electric field effect has also been demonstrated to activate intensive lymphocytic and dendritic cell penetration into tumor (13).

The abscopal effect from radiotherapy (RT) has been known for a long time and was interpreted as an immune-mediated effect (14). Despite millions of patients having been treated with RT, only 46 abscopal cases induced by radiation treatment alone have been described between 1969 and 2014 (15). In preclinical models, the combination of immune check point (ICP) inhibitors with RT has demonstrated abscopal effects, but human reports still remain sparse, largely restricted to melanoma and nonsmall cell lung cancer (16-21). However, in all such reports, it remains difficult to ascribe the abscopal effect purely to RT alone or in combination with immune enhancing therapeutics (22). A prospective trial of RT, with a granulocyte-macrophage colony stimulating factor in metastatic diseases, reported a surprisingly high abscopal effect of $27.6 \%$ (23). RT creates tumor and normal tissue damage, lysis, and antigen release for sustained in-vivo vaccination events. Thymus-derived regulatory $\mathrm{T}$ (Treg) cells played a critical role in the control of immune tolerance to self-antigens, however, they also resulted in reduced antitumor immunity (24). There were very few literature reports on how therapy related autoimmunity-mediated antitumor activity $(25,26)$.

We speculated that the incidence of the abscopal effect may be higher in patients who develop autoimmunity. Bakacs et al. reported that immune related adverse events (irAEs) induced by ipilimumab are very similar to the chronic graft vs. host disease that ensues allogenic bone marrow transplantation (27). Autoreactive $\mathrm{T}$ cells may bypass the negative selection pressure in the microenvironment of the tumor and differentiate to memory $\mathrm{T}$ cells that recognize both "self" and "tumor." We report, we believe for first time, that patients treated with RT and mEHT may have a long treatment-free period once they unleash an autoimmune reaction, and further, that in such patients, successful salvaging through low-dose ICP inhibitors may be possible at tumor recurrence.

\section{MATERIALS AND METHODS}

We performed a single institution, observational case-cohort study for patients with metastatic cancers of various origins, treated with a combination of RT and $\mathrm{mEHT}$, with a minimum RT dose of $30 \mathrm{~Gy}$ and at least four mEHT treatments, to report unexpected adverse events. This retrospective analysis was conducted as part of a post-marketing safety surveillance program after the approval of the $\mathrm{mEHT}$ device in the class III medical category in Taiwan. The study was approved by the Institutional Review Board and was conducted according to the guidelines of Good Clinical Practice.

\section{Patient Selection}

Enrolled patients were 20 years of age or older, presented with inoperable, recurrent, or metastatic diseases, requiring palliation with RT. In our study, all patients underwent concurrent RT and mEHT with or without systemic therapies, based on the underlying clinical condition. All institution-specific consent requirements were adhered to; written informed consent was obtained from the participants for the publication of the case series.

\section{Radiotherapy}

RT was performed using conventional fractionation (and not hypofractionated) schedules, with a dose of 2 to $3.5 \mathrm{~Gy}$ per fraction (fx), five times per week to at least $30 \mathrm{~Gy}$, as clinically appropriate and necessary. The clinical target volume (CTV) was defined as the gross tumor volume (GTV) plus a margin of 3-5 mm, based on the specific tumor type being addressed. Patients were treated with Elekta Synergy ${ }^{\circledR}$ (Elekta, Stockholm, Sweden) or TomoTherapy ${ }^{\circledR}$ (Accuray, Sunnyvale, CA, USA) with standard immobilization devices, using image-guided, modulated arc therapy with 6-MV photons for most of the patients. For patients who had received RT prior to the study, the original treatment plans were retrieved in every case of suspected overlap with the prior RT fields, and appropriate organ-at-risk constraints were adhered to. 


\section{Hyperthermia (mEHT)}

The mEHT treatment was applied using an EHY 2000+ hyperthermia device (OncoTherm GmbH, Germany). Treatment lasted for $60 \mathrm{~min}$ and was administered once weekly. A $30 \mathrm{~cm}$ in diameter circular electrode was placed at the irradiated tumor site, approximating placement at the radiation field isocenter. A $13.56 \mathrm{MHz}$ radiofrequency (RF) was used with a real-time, automatic tuning device resulting in energy-transfer matching and ensuring a standard wave ratio of $\sim 1$ (the most ideal value). The power was initially set to 80 Watts (W) and a step-up protocol was applied to increase by $20-30 \mathrm{~W}$ every $5 \mathrm{~min}$, until $150 \mathrm{~W}$ was reached for the remaining treatment duration. The goal for the target energy delivered was minimally set at $500 \mathrm{~kJ}$ per treatment. All appropriate vital sign monitoring during and after treatment was conducted as per standard practice. With this technique, intratumoral temperature measurement is typically not performed because the temperature elevation measured by a conventional thermocouple is usually $<2^{\circ} \mathrm{C}$ (28). Adverse events were assessed throughout each treatment, which included heat sensitivity, skin burning, pain, and gastric discomfort.

\section{Outcomes Evaluation}

The primary endpoint was toxicity, which was evaluated weekly and recorded using the Common Terminology Criteria for Adverse Events (CTCAE) version 4.0. during the RT and mEHT period and 2 months after. The secondary outcome was the radiologic response, which was evaluated on the irradiated lesions according to the Response Evaluation Criteria in Solid Tumors (RECIST) version 1.1 (29) every 3 months with CT, PET-CT, or tumor markers, based on a baseline selection diagnostic/imaging finding. The response categories of interest included complete response (CR), partial response (PR), stable disease (SD), and progressive disease (PD). Most patients received some kind of systemic treatment afterward. The length of follow-up was defined from the last day of RT to the last followup visit. Baseline measurements and changes in the neutrophil to lymphocyte ratio (N/L) before and after treatment were collected.

\section{Statistical Analysis}

The impact of patient-, tumor-, and treatment-related factors on response was evaluated using a univariate and multivariable analysis. Survival curves were estimated using the KaplanMeier method. Fisher's exact test (two-tailed test) was used for evaluating $2 \times 2$ tables for significance. Statistical analyses were performed with the SAS statistical software (version 9.2; SAS Institute, Cary, NC, USA). $P<0.05$ were set for statistical significance.

\section{RESULTS}

\section{Patient and Disease Characteristics}

Thirty-three patients with recurrent or metastatic cancer was enrolled between January 2017 and December 2017. Patient characteristics are listed in Table 1. The median patient age was 59.3 years (range, 38-84 years). Breast cancer, lung cancer, hepatoma, cholangiocarcinoma, and urothelial carcinoma were the five most common disease entities. The thorax was the most
TABLE 1 | Patient characteristics.

\begin{tabular}{|c|c|c|}
\hline Characteristics & No. & $\%$ \\
\hline \multicolumn{3}{|l|}{ Sex } \\
\hline Female & 17 & 51.5 \\
\hline Male & 16 & 48.5 \\
\hline Age, median, range, years & 59.4 & 38-84 years-old \\
\hline \multicolumn{3}{|l|}{ Disease entities } \\
\hline Breast cancer & 8 & 24.4 \\
\hline Lung cancer & 4 & 12.1 \\
\hline Hepatocellular carcinoma & 3 & 9 \\
\hline Cholangiocarcinoma & 3 & 9 \\
\hline Urothelial carcinoma & 3 & 9 \\
\hline Others & 12 & 36.5 \\
\hline \multicolumn{3}{|l|}{ Treatment before RT+ mEHT } \\
\hline Surgery & 12 & 36.3 \\
\hline RT & 19 & 57.6 \\
\hline ChT (include hormone, target therapy) & 21 & 63.6 \\
\hline 10 & 0 & 0 \\
\hline $\mathrm{Cht}+\mathrm{IO}$ & 2 & 6 \\
\hline \multicolumn{3}{|l|}{ Treatment during RT+ mEHT } \\
\hline ChT (include hormone, target therapy) & 16 & 48.5 \\
\hline 10 & 9 & 27.2 \\
\hline $\mathrm{ChT}+\mathrm{IO}$ & 6 & 18.2 \\
\hline \multicolumn{3}{|l|}{ Treatment after RT+ mEHT } \\
\hline Surgery & 1 & 4.6 \\
\hline ChT (include hormone, target therapy) & 13 & 59.1 \\
\hline 10 & 3 & 13.6 \\
\hline $\mathrm{ChT}+\mathrm{IO}$ & 5 & 22.7 \\
\hline
\end{tabular}

Cht, chemotherapy; IO, immune-Oncology.

commonly treated site (12 patients in total, including four lung cancer patients and eight for breast cancer) followed by abdomen (nine patients) and liver (six patients). During the RT and mEHT treatment, 16 patients received concurrent chemotherapy, nine had ICP inhibitors and six were treated with both agents. The median follow-up time was 11.6 months (range, 4-22.7 months) with no patients lost for follow-up.

\section{Treatment}

The median RT dose was 45.5 Gy (range, 30-66 Gy), and the mean GTV was $138.9 \mathrm{~cm}^{3}$ (ranged between 20 and 5064.7 $\mathrm{cm}^{3}$ ). The median number of mEHT treatment fractions was 12 (range, 4-20).

\section{Treatment Outcome and Toxicities}

The combination of RT and mEHT treatment was well-tolerated. A full listing of adverse events during the RT plus mEHT treatment is provided in Table 2. Common treatment-related adverse events were grade 1 skin, and grade 2 myelotoxicities. Transient core body temperature elevation $\left(>38^{\circ} \mathrm{C}\right)$, which resolved shortly after mEHT treatment, was noted in six patients; two obese patients had localized subcutaneous fat induration that persisted for several weeks, and then resolved. The most important adverse events that went beyond our expectation were 
TABLE 2 | Treatment toxicities during RT + mEHT.

\begin{tabular}{|c|c|c|}
\hline \multicolumn{3}{|c|}{ Treatment toxicity (CTCAE v4.0) } \\
\hline Toxicity & Case number $(N)$ & $\%$ \\
\hline \multicolumn{3}{|c|}{ Skin toxicity } \\
\hline Grade 0 & 20 & 60.6 \\
\hline Grade 1 & 12 & 36.3 \\
\hline Grade 2 & $1^{*}$ & 3.1 \\
\hline Grade 3 & 0 & 0 \\
\hline \multicolumn{3}{|c|}{ Hepatic toxicity } \\
\hline Grade 0 & 31 & 93.8 \\
\hline Grade 1 & 1 & 3.1 \\
\hline Grade 2 & 0 & 0 \\
\hline Grade 3 & $1^{\text {** }}$ & 3.1 \\
\hline \multicolumn{3}{|c|}{ Myelotoxicity } \\
\hline Grade 0 & 24 & 72.7 \\
\hline Grade 1 & 1 & 3.1 \\
\hline Grade 2 & 6 & 18.2 \\
\hline Grade 3 & 2 & 6.0 \\
\hline \multicolumn{3}{|c|}{ Neurotoxicity } \\
\hline Grade 0 & 32 & 96.6 \\
\hline Grade 1 & 0 & 0 \\
\hline Grade 2 & 0 & 0 \\
\hline Grade 3 & $1^{\star \star \star}$ & 3.1 \\
\hline \multicolumn{3}{|c|}{ Nausea and vomiting } \\
\hline Grade 0 & 30 & 90.9 \\
\hline Grade 1 & 1 & 3.1 \\
\hline Grade 2 & 2 & 6.0 \\
\hline Grade 3 & 0 & 0 \\
\hline \multicolumn{3}{|l|}{ Diarrhea } \\
\hline Grade 0 & 30 & 90.9 \\
\hline Grade 1 & 1 & 3.1 \\
\hline Grade 2 & 2 & 6.0 \\
\hline Grade 3 & 0 & 0 \\
\hline \multicolumn{3}{|c|}{ Elevated core body temperature (after treatment) } \\
\hline Yes & 6 & 18.2 \\
\hline No & 27 & 81.8 \\
\hline \multicolumn{3}{|c|}{ Fat induration } \\
\hline Yes & 2 & 6.0 \\
\hline No & 31 & 94.0 \\
\hline
\end{tabular}

${ }^{*}$ Autoimmune reaction: Dermatitis herpetiformis.

${ }^{\star \star}$ Autoimmune reaction: Autoimmune hepatitis.

${ }^{\star \star \star}$ Autoimmune reaction: Myasthenia gravis.

autoimmune related toxicities (three out of 33 patients, 9.1\%). One patient treated only with RT and mEHT developed grade 3 autoimmune hepatitis. One patient who was simultaneously treated with low-dose ICP (Yervoy ${ }^{\circledR}$ and Opdivo ${ }^{\circledR}$ ) developed grade 3 myasthenia gravis, and another patient developed grade 2 autoimmune-related skin toxicity (dermatitis herpetiformis). All three patients who developed autoimmune toxicities had long lasting abscopal effects in the absence of any further subsequent systemic treatment.

Among the in-field evaluable lesions treated with RT and mEHT (39 lesions in 33 patients), CR, PR, SD, and PD were observed in 6.1, 54.5, 27.3, and $12.1 \%$ of patients (Table 3). All eight breast cancer patients had $\geqq P R$ response. Somewhat surprisingly, larger tumors $(>500 \mathrm{ml}$ ) demonstrated superior responses than smaller tumors $(<500 \mathrm{ml})(100$ vs. $48 \%, p=$ 0.012) (Table 3). All the patients with autoimmune toxicities had a tumor size of more than $500 \mathrm{ml}$. Because of the small sample size, multivariate analysis failed to show significant differences between response and age, tumor size, number of mEHT treatments, tumor depth, the use of ICP inhibitors, chemotherapy, and autoimmune reactions. Thirteen patients (39.4\%) had a decreased N/L ratio 1 month after $\mathrm{RT}+\mathrm{mEHT}$, which includes two patients with $\mathrm{CR}$, six with PR, three with SD and two with PD (Table 3). The three patients with an autoimmune abscopal effect had an elevated N/L ratio before treatment $(>8)$ which decreased to $<3.5$ after treatment. The median survival time was 11.4 months (range, 2.6-16.9 months) in patients whose $\mathrm{N} / \mathrm{L}$ decreased, vs. 8.9 months in patients with elevated N/L (range, 1.7-16.2 months).

\section{Case Presentation: Autoimmune Phenomena Associated With Abscopal Tumor Response} Case 1

A 42-year-old female patient presented with a left breast ulcerative fungating mass $(>10 \mathrm{~cm})$ with palpable bilateral axillary lymph nodes. She was diagnosed with metastatic, left breast, triple-negative invasive ductal carcinoma. She refused chemotherapy and received palliative RT consisting of $50 \mathrm{~Gy}$ in 25 fxs plus weekly mEHT for six treatments. Elevated serum alanine and aspartate aminotransferases (ALT and AST), alkaline phosphatase and bilirubin was identified 2 weeks after RT. Positive anti-microsomal antibody and anti-smooth muscle antibody levels assisted in making a diagnosis of autoimmune hepatitis. She was treated with prednisone (starting at $40 \mathrm{mg}$ daily and tapered to $10 \mathrm{mg}$ daily within 4 weeks). The primary tumor shrank rapidly to $\sim 1 \mathrm{~cm} 1$ month after treatment and a wide excision was performed 2 months later (Figure 1A). The bilateral axillary and the left internal mammary metastatic lymph nodes outside the local treatment field demonstrated dramatic and sustained regression, qualifying for our abscopal response criteria. More than 1 year later, she developed lung metastases and was treated bi-weekly with reduced-dose ICP inhibitor treatment ( $60 \mathrm{mg}$ of Opdivo $\left.{ }^{\circledR}\right)$ for two doses with a significant response (Figure 1B), resulting in a CR. Subsequently, her serum AST, ALT, and bilirubin levels increased once again, suggesting relapse of her autoimmune hepatitis, resulting in discontinuation of immunotherapy (Figure 1C). Despite this, her lung metastases demonstrated sustained remission, and she is still alive and tumor-free, $>12$ months after discontinuing ICP therapy.

\section{Case 2}

A 60-year-old female had right renal pelvis urothelial carcinoma diagnosed in October 2016. She underwent robotic right nephroureterectomy and bladder cuff excision, revealing a pT4N0 tumor treated with adjuvant tumor bed radiotherapy to $48 \mathrm{~Gy}$ in 24 fxs (completed in January 2017). In May 2017, she presented with a rapidly growing, painful, palpable abdominal mass. An abdominal CT scan showed multiple intra-abdominal 
TABLE 3 | Response rate of the irradiated sites.

\begin{tabular}{|c|c|c|c|c|c|}
\hline Response & $\begin{array}{l}\text { Metastatic/recurrent } \\
\qquad(N=33)\end{array}$ & $\begin{aligned} & \text { GTV } \geq 500 \mathrm{ml} \\
&(N=8)^{\star *}\end{aligned}$ & $\begin{array}{c}\text { GTV }<500 \mathrm{ml} \\
\quad(N=25)\end{array}$ & $\begin{array}{l}\text { Decreased } N / L \text { ratio post } \\
\text { treatment }(N=13)^{\star \star \star \star}\end{array}$ & $\begin{array}{c}\text { Increased } N / L \text { ratio post } \\
\text { treatment }(N=20)\end{array}$ \\
\hline CR & 2 (6.1\%) & \multirow{3}{*}{$8^{\star \star \star}(100 \%)$} & \multirow{3}{*}{12 (48\%) } & 2 & 0 \\
\hline VGPR $^{*}$ & 5 (15.2\%) & & & 3 & 2 \\
\hline PR & 13 (39.4\%) & & & 3 & 10 \\
\hline SD & 9 (27.3\%) & \multirow{2}{*}{0 (0\%) } & \multirow{2}{*}{13 (52\%) } & 3 & 6 \\
\hline PD & $4(12.1 \%)$ & & & 2 & 2 \\
\hline
\end{tabular}

*VGPR, Very good partial response defined as $>90 \%$ regression.

${ }^{* *} p=0.012$ Fisher's exact test.

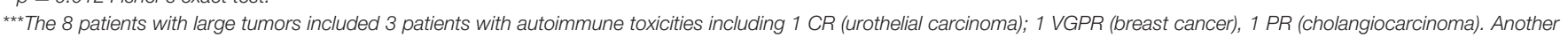
5 patients included 1VGPR (hepatoma) and 4 PR (1 cervix and 3 breast cancers).

${ }^{\star \star \star \star} p=0.245$ Fisher's exact test.

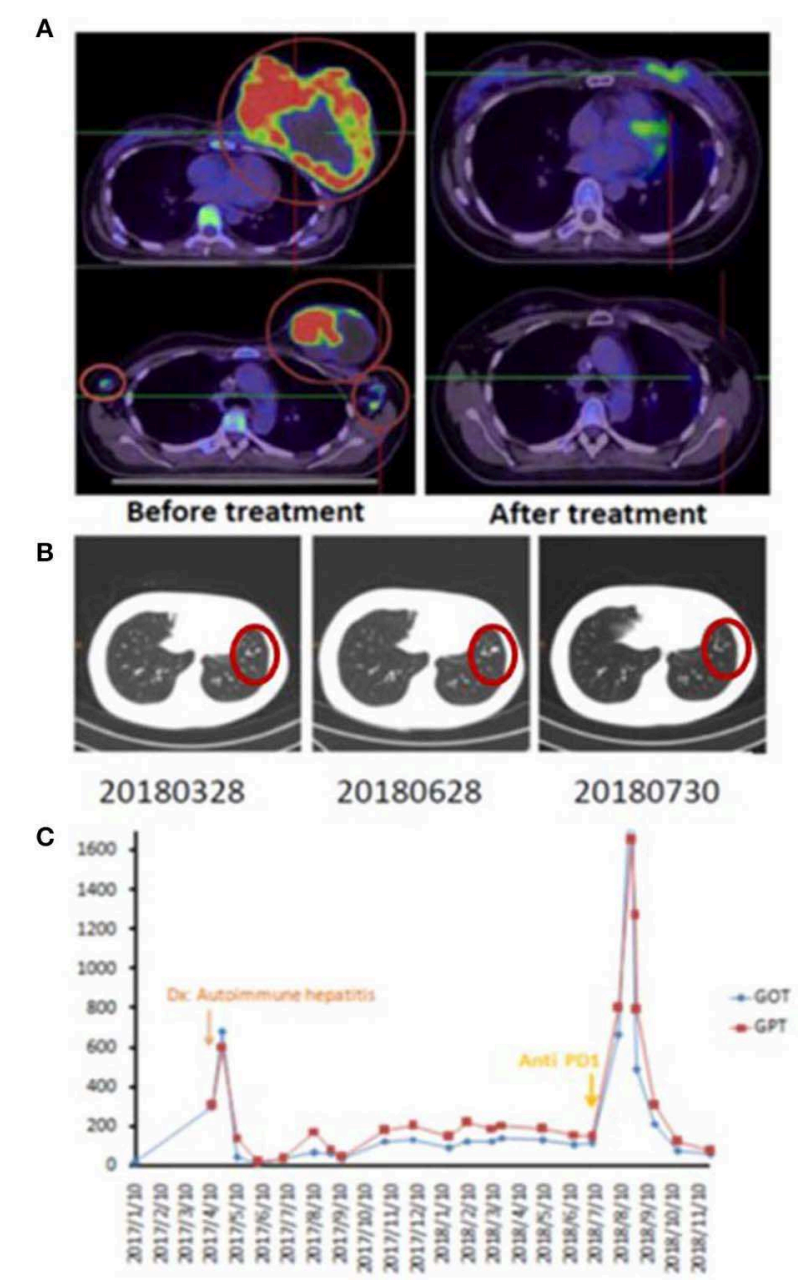

FIGURE 1 | Representative patient (Case 1) with autoimmune mediated abscopal effects. (A) Locally advanced breast cancer with tumor abscopal effect on bilateral axillary and internal mammary lymph nodes. (B) Progressive lung metastatic lesions successfully salvaged with 2 cycles of low dose Opdivo ${ }^{\circledR}$. (C) Flare up of autoimmune hepatitis by RT plus mEHT and ICP inhibitors. masses and a right retroperitoneal mass attached to the right psoas muscle. The largest tumor was $4.5 \mathrm{~cm}$. There was also a separate lower anterior abdominal wall mass and a liver segment 7 metastases. She received a second course of palliative RT targeting the symptomatic and dominant right lower quadrant mass and the lower abdominal wall mass, both treated to $40 \mathrm{~Gy}$ in $20 \mathrm{fxs}$, along with five weekly mEHT treatments. Concomitant carboplatin at $300 \mathrm{mg}$ and gemcitabine at $600 \mathrm{mg}$ were given for only two cycles and discontinued after pancytopenia developed. The abdominal pain resolved quickly, and she developed a mild fever with elevated CRP and pancytopenia in the 3rd week of treatment. A generalized itchy skin rash developed over the trunk in the 4th week of treatment. She was diagnosed with dermatitis herpetiformis and macrocytic anemia with positive anti-parietal cell antibody. The skin lesions were controlled with low-dose prednisolone (10 mg, once daily). A CT scan in August 2017 showed CR at the irradiated sites. Unexpectedly, an abscopal effect of the hepatic metastases was also identified (Figure 2) which was unlikely to be from the systemic effect of only two cycles of low doses of carboplatin and gemcitabine. No further treatment was administered. A recent follow-up CT scan in May 2019, 2 years after palliative RT, showed persistent CR of all disease sites.

\section{Case 3}

This 69-year-old male patient had a biopsy-proven cholangiocarcinoma with multiple metastases diagnosed in August 2017. He began treatment with immunotherapy (Yervoy ${ }^{\circledR}$ at $50 \mathrm{mg}$ for one dose only and Opdivo ${ }^{\circledR}$ at $60 \mathrm{mg}$ every 2 weeks) for eight cycles, RT (45 Gy in 15 fxs to the liver, $30 \mathrm{~Gy}$ in $10 \mathrm{fxs}$ to the scapula, L1 spine, and right pelvic bone), and weekly mEHT for 12 sessions starting from September 2017. In December 2017, he suffered from progressive muscle weakness with mild ptosis, lethargy, and difficulty in swallowing. He developed an aspiration pneumonia, requiring intubation and supportive management in the neurology intensive care unit. A positive acetylcholine receptor (AchR) antibody with electromyogram findings confirmed a new diagnosis of myasthenia gravis (MG). The patient gradually recovered after 


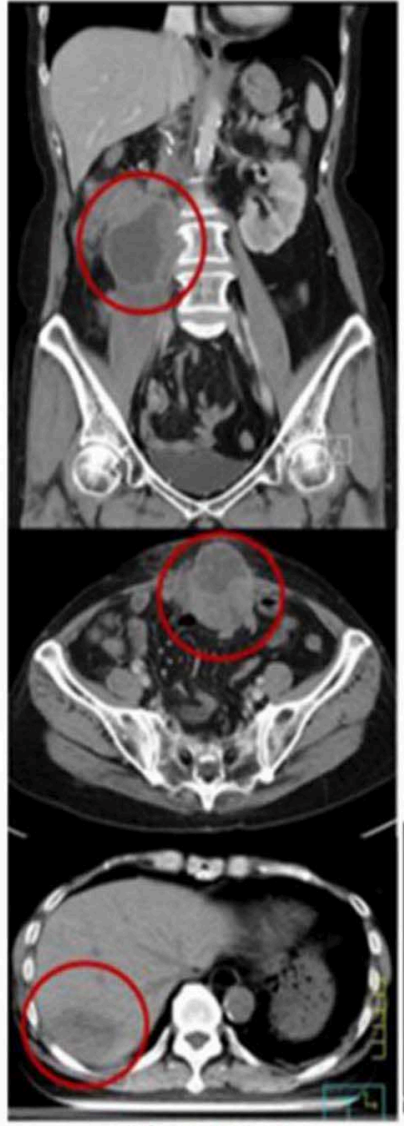

Before treatment

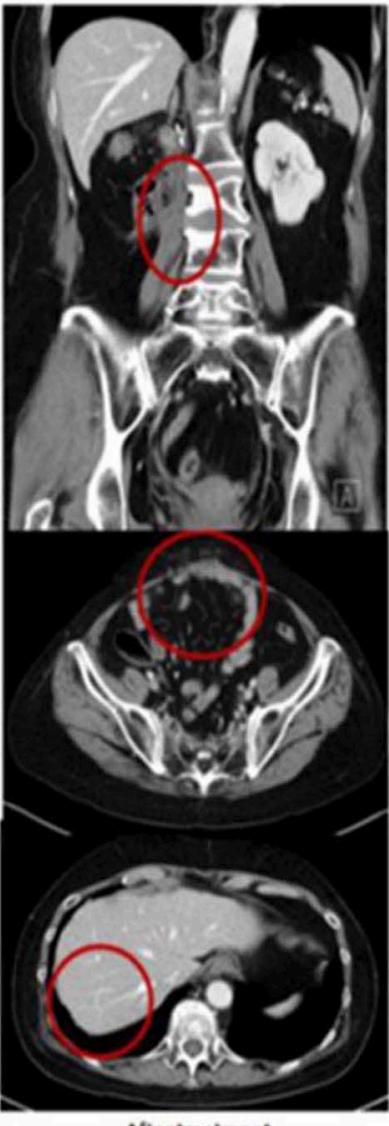

Aftertreatment

FIGURE 2 | Representative patient (Case 2) with autoimmune mediated abscopal effects: metastatic urothelial carcinoma with abscopal tumor effect on liver metastases.

receiving plasmapheresis, steroids, and antibiotics. He did not receive any further anti-neoplastic therapy and was maintained on prednisolone, $5 \mathrm{mg}$ once daily, for the subsequent 10 months. Follow-up imaging showed good PR at irradiated sites with measurable PR of the unirradiated L5 spine metastases. The CA 19-9 level peaked to $555 \mathrm{U} / \mathrm{ml}$ in $2018 / 3$ and gradually dropped to $76.7 \mathrm{U} / \mathrm{ml}$ in $2019 / 6$ (Figure 3) and he remained asymptomatic without any systemic treatment.

\section{DISCUSSION}

Although our patients were heterogeneous in terms of histology, lesion numbers, and prior treatments, in general, they represented a relatively common pool of patients referred for palliative radiotherapy, i.e., relatively large, symptomatic disease, either heavily pretreated or having declined during other therapies. In that context, the $60.6 \%$ overall response rates of the locally treated (RT plus mEHT) lesions may suggest a synergistic or radiosensitizing effect. Unexpectedly, tumors larger than $500 \mathrm{ml}$, had an even better response rate. Intriguingly, three cases of autoimmunity occurred after treatment, which was associated with abscopal tumor response.

What possible mechanisms could be at play here? The combination of systemic autoimmune effects and tumor abscopal effects provoked by combined $\mathrm{mEHT}$ and RT from local treatment leads us to speculate the possibility of the clonal expansion of a subset of $\mathrm{T}$ cells targeting both tumor antigens and shared normal tissue epitopes. These patients required steroids for managing their autoimmune reactions, without loss of tumor control. mEHT induces tumor cytotoxicity through a combination of localized thermal effects, and the temperature independent signal-excitation effect for DAMP release (12, 30). HSP-associated DAMP could facilitate immunogenicity, especially in the context of concomitant RT and possible combination with immune checkpoint inhibitors. The addition of ICP inhibitors after concomitant chemoradiotherapy (CCRT) in stage III lung cancer patients, improves both progressionfree and overall survival rates relative to any other consolidative approach, suggesting the possibility that localized therapy creates a milieu for ICPs to have more durable effects $(31,32)$.

Are the local and abscopal responses reported herein, especially their depth and durability, expected and routine? Patients in this study were generally at such an advanced stage of their disease, that first, the expected response rates would be rather low, and second, durability would be very uncommon. This leads us to hypothesize that our clinical observations would require the development of unleashed anti-tumor autoimmunity, possibly from combinatorial $\mathrm{mEHT}$ and RT. In the three cases with autoimmune toxicity, Case 1 was treated with RT and mEHT only; Case 2 received only two cycles of reduced dose concomitant chemotherapy and Case 3 had immunotherapy with RT. It would be very unlikely that the abscopal liver metastasis response in Case 2 was a chemotherapy effect. Whether the remote bony metastases response in Case 3 qualified as a pure "abscopal effect" is debatable. Nevertheless, the autoimmune reactions in the three cases after local treatment were quite clear. Immune response through in-situ vaccination might be amplified by the addition of ICP inhibitors as the third case described or might yield a deeper response as the second case described. Gauci et el. recently reported that in order to prolong survival, a CR or PR within 3 months after treatment was mandatory with anti-PD-(L)1 monotherapy for multiple cancer types (33).

Larger tumors had better responses to combined RT and mEHT treatment, which is counterintuitive. Explanations for this include the possibility that large tumors, especially those near the body surface under the electrode, absorb more energy from the RF current (34). For example, in an in vivo experiment, the use of a large $20 \mathrm{~mm}$ diameter electrode to deliver $\mathrm{mEHT}$ to $8 \mathrm{~mm}$ diameter size murine tumors resulted in impressive apoptosis, necrosis, and extracellular damageassociated molecular secretion patterns $(12,13)$, presumably because the entirety of the tumor was able to absorb energy effectively. Similarly, all eight breast cancers responded to this treatment. The radiation fractional dose used in our series is classic and typical for palliative radiotherapy, but atypical as far as several preclinical combinatorial immune checkpoint-radiation experiments recommend (for example 5-9 Gy per fraction, 3-5 fractions). Despite the use of lower fractional doses, the responses observed herein are robust. This could reflect the combinatorial use of mEHT. However, it is also worth considering that other 


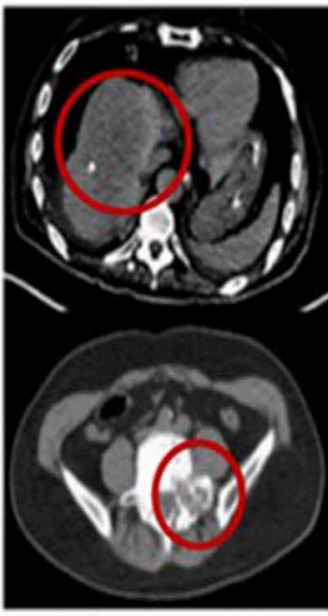

Before treatment

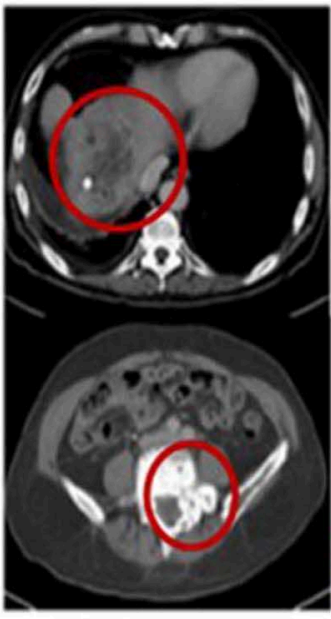

After treatment

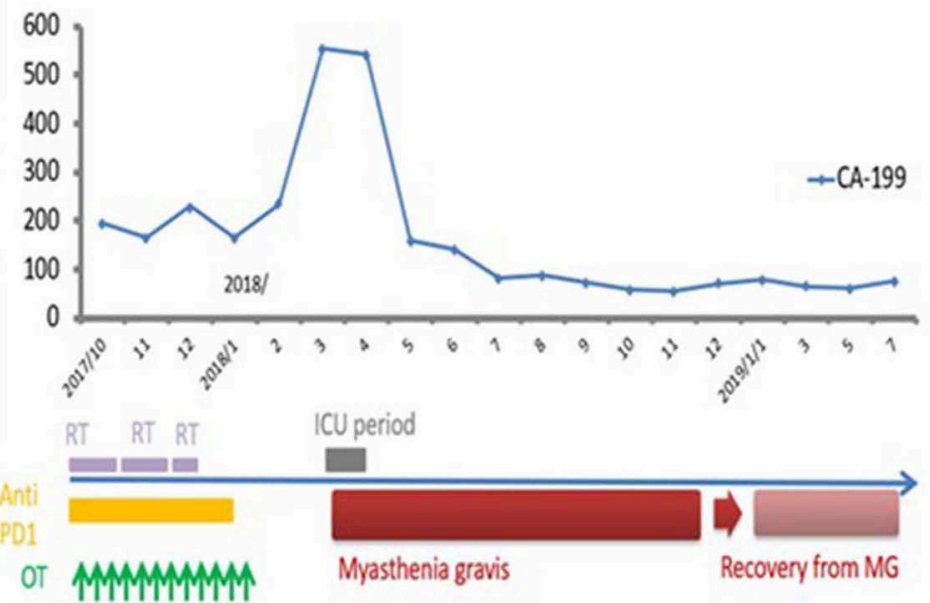

FIGURE 3 | Representative patient (Case 3) with autoimmune mediated abscopal effects: metastatic cholangiocarcinoma with abscopal tumor effect on L5 bony metastases and change of CA-199 level.

clinical reports, such as the one by Chandra et al., demonstrated that radiation fraction size $<3 \mathrm{~Gy}$ was the only parameter identified to be associated with favorable index lesion response in a cohort of melanoma patients treated with immune checkpoint inhibitors and radiotherapy (20).

As the use of immunotherapy becomes more popular, irAE is emerging as an issue (35). irAEs from ICP inhibitors are generally regarded as a "toxicity," however, a number of reports are beginning to appear in the literature claiming that patients with higher irAEs may have a higher response rate $(36,37)$. In addition, patients with irAE have longer treatment durations and more time to develop autoimmune toxicities (35). Clearly, immunotoxicity and autoimmunity is a balancing act. In our study, three out of 33 patients $(9.1 \%)$ had induced autoimmune reactions from $\mathrm{RT}+\mathrm{mEHT}$. They all had a profound abscopal effect ( $>90 \%$ shrinkage of non-irradiated tumor, lasting for more than 12 months) without any substantial systemic targeted, cytotoxic, or ICP inhibitor therapy when autoimmune toxicities were noted. Most abscopal effects reported in the literature are neither "deep" (i.e., $>90 \%$ tumor reduction), nor durable. For example, in the series by Golden et al. (23), only two of 41 patients (4.9\%) had a dramatic abscopal response according to the criteria of $>90 \%$ tumor reduction. Therefore, we argue that the effective immunity may be coupled to autoimmunity.

This case series of combining $\mathrm{mEHT}$ and RT for palliative purposes demonstrated unexpected autoimmune toxicities along with dramatic and sustained tumor regression. Despite being interesting and inspiring, these results must be interpreted

\section{REFERENCES}

1. Datta NR, Ordonez SG, Gaipl US, Paulides MM, Crezee H, Gellermann J, et al. Local hyperthermia combined with radiotherapy and-/or chemotherapy: recent advances and promises for the future. Cancer Treat Rev. (2015) 41:74253. doi: 10.1016/j.ctrv.2015.05.009 with great caution and at best provide initial observations for hypothesis-generation, as there are considerable limitations given the retrospective, single institution analysis, with limited patient numbers, and considerable heterogeneity. An official prospective trial combining immune check point inhibitors with RT and mEHT will be launched.

\section{DATA AVAILABILITY STATEMENT}

All datasets generated for this study are included in the article/supplementary material.

\section{ETHICS STATEMENT}

The studies involving human participants were reviewed and approved by Institute Review Board of Shin Kong Wu Ho Su Memorial Hospital. Written informed consent for participation was not required for this study in accordance with the national legislation and the institutional requirements.

\section{AUTHOR CONTRIBUTIONS}

M-SC, MM, and K-HC: conception and design and writing review of the manuscript. K-LY, H-CL, Y-SW, H-LK, Y-CL, and K-HC: development of methodology. M-SC: acquisition of data and analysis and interpretation of data (e.g., statistical analysis, biostatistics, computational analysis). K-HC: study supervision. All authors read and approved the final manuscript.

2. Minnaar CA, Kotzen JA, Ayeni OA, Naidoo T, Tunmer M, Sharma V, et al. The effect of modulated electro-hyperthermia on local disease control in HIV-positive and -negative cervical cancer women in South Africa: early results from a phase III randomised controlled trial. PLoS ONE. (2019) 14:e0217894. doi: 10.1371/journal.pone. 0217894 
3. Lee SY, Szigeti GP, Szasz AM. Oncological hyperthermia: the correct dosing in clinical applications. Int J Oncol. (2019) 54:627-43. doi: 10.3892/ijo.2018.4645

4. Fiorentini G, Szasz A. Hyperthermia today: electric energy, a new opportunity in cancer treatment. J Cancer Res Ther. (2006) 2:41-6. doi: 10.4103/0973-1482.25848

5. Kirson ED, Gurvich Z, Schneiderman R, Dekel E, Itzhaki A, Wasserman Y, et al. Disruption of cancer cell replication by alternating electric fields. Cancer Res. (2004) 64:3288-95. doi: 10.1158/0008-5472.CAN-04-0083

6. Jo Y, Hwang SG, Jin YB, Sung J, Jeong YK, Baek JH, et al. Selective toxicity of tumor treating fields to melanoma: an in vitro and in vivo study. Cell Death Discov. (2018) 4:46. doi: 10.1038/s41420-018-0106-x

7. Papp E, Vancsik T, Kiss E, Szasz O. Energy absorption by the membrane rafts in the modulated electro-hyperthermia (mEHT). Open J Biophys. (2017) 7:216-29. doi: 10.4236/ojbiphy.2017.74016

8. Cha J, Jeon TW, Lee CG, Oh ST, Yang HB, Choi KJ, et al. Electrohyperthermia inhibits glioma tumorigenicity through the induction of E2F1-mediated apoptosis. Int J Hyperthermia. (2015) 31:784-92. doi: 10.3109/02656736.2015.1069411

9. Yang KL, Huang CC, Chi MS, Chiang HC, Wang YS, Hsia CC, et al. In vitro comparison of conventional hyperthermia and modulated electrohyperthermia. Oncotarget. (2016) 7:84082-92. doi: 10.18632/oncotarget.11444

10. Jeon TW, Yang H, Lee CG, Oh ST, Seo D, Baik IH, et al. Electrohyperthermia up-regulates tumour suppressor Septin 4 to induce apoptotic cell death in hepatocellular carcinoma. Int J Hyperthermia. (2016) 32:648-56. doi: 10.1080/02656736.2016.1186290

11. Yang W, Han GH, Shin HY, Lee EJ, Cho H, Chay DB, et al. Combined treatment with modulated electro-hyperthermia and an autophagy inhibitor effectively inhibit ovarian and cervical cancer growth. Int J Hyperthermia. (2019) 36:9-20. doi: 10.1080/02656736.2018.1528390

12. Andocs G, Meggyeshazi N, Balogh L, Spisak S, Maros ME, Balla P, et al. Upregulation of heat shock proteins and the promotion of damageassociated molecular pattern signals in a colorectal cancer model by modulated electrohyperthermia. Cell Stress Chaperones. (2015) 20:37-46. doi: 10.1007/s12192-014-0523-6

13. Tsang YW, Huang CC, Yang KL, Chi MS, Chiang HC, Wang YS, et al. Improving immunological tumor microenvironment using electrohyperthermia followed by dendritic cell immunotherapy. BMC Cancer. (2015) 15:708. doi: 10.1186/s12885-015-1690-2

14. Wang R, Zhou T, Liu W, Zuo L. Molecular mechanism of bystander effects and related abscopal/cohort effects in cancer therapy. Oncotarget. (2018) 9:18637-47. doi: 10.18632/oncotarget.24746

15. Abuodeh Y, Venkat P, Kim S. Systematic review of case reports on the abscopal effect. Curr Probl Cancer. (2016) 40:25-37. doi: 10.1016/j.currproblcancer.2015.10.001

16. Deng L, Liang H, Burnette B, Beckett M, Darga T, Weichselbaum RR, et al. Irradiation and anti-PD-L1 treatment synergistically promote antitumor immunity in mice. J Clin Invest. (2014) 124:687-95. doi: 10.1172/JCI67313

17. Twyman-Saint Victor C, Rech AJ, Maity A, Rengan R, Pauken KE, Stelekati E, et al. Radiation and dual checkpoint blockade activate nonredundant immune mechanisms in cancer. Nature. (2015) 520:373-7. doi: $10.1038 /$ nature 14292

18. Bernstein MB, Krishnan S, Hodge JW, Chang JY. Immunotherapy and stereotactic ablative radiotherapy (ISABR): a curative approach? Nat Rev Clin Oncol. (2016) 13:516-24. doi: 10.1038/nrclinonc.2016.30

19. Bhalla N, Brooker R, Brada M. Combining immunotherapy and radiotherapy in lung cancer. J Thorac Dis. (2018) 10(Suppl 13):S1447-S60. doi: $10.21037 /$ jtd.2018.05.107

20. Chandra RA, Wilhite TJ, Balboni TA, Alexander BM, Spektor A, Ott $\mathrm{PA}$, et al. A systematic evaluation of abscopal responses following radiotherapy in patients with metastatic melanoma treated with ipilimumab. Oncoimmunology. (2015) 4:e1046028. doi: 10.1080/2162402X.2015.1046028

21. Bitran J. The abscopal effect exists in non-small cell lung cancer: a case report and review of the literature. Cureus. (2019) 11:e4118. doi: 10.7759/cureus.4118

22. Ngwa W, Irabor OC, Schoenfeld JD, Hesser J, Demaria S, Formenti SC. Using immunotherapy to boost the abscopal effect. Nat Rev Cancer. (2018) 18:313-22. doi: $10.1038 / \mathrm{nrc} .2018 .6$
23. Golden EB, Chhabra A, Chachoua A, Adams S, Donach M, FentonKerimian $\mathrm{M}$, et al. Local radiotherapy and granulocyte-macrophage colonystimulating factor to generate abscopal responses in patients with metastatic solid tumours: a proof-of-principle trial. Lancet Oncol. (2015) 16:795-803. doi: 10.1016/S1470-2045(15)00054-6

24. Galgani M, Di Giacomo A, Matarese G, La Cava A. The yin and yang of $\mathrm{CD} 4(+)$ regulatory $\mathrm{T}$ cells in autoimmunity and cancer. Curr Med Chem. (2009) 16:4626-31. doi: 10.2174/092986709789878201

25. Sultan H, Trillo-Tinoco J, Rodriguez P, Celis E. Effective antitumor peptide vaccines can induce severe autoimmune pathology. Oncotarget. (2017) 8:70317-31. doi: 10.18632/oncotarget.19688

26. Miska J, Bas E, Devarajan P, Chen Z. Autoimmunity-mediated antitumor immunity: tumor as an immunoprivileged self. Eur J Immunol. (2012) 42:2584-96. doi: 10.1002/eji.201242590

27. Bakacs T, Mehrishi JN. Anti-CTLA-4 therapy may have mechanisms similar to those occurring in inherited human CTLA4 haploinsufficiency. Immunobiology. (2015) 220:624-5. doi: 10.1016/j.imbio.2014. 11.019

28. Lee SY, Kim JH, Han YH, Cho DH. The effect of modulated electrohyperthermia on temperature and blood flow in human cervical carcinoma. Int J Hyperthermia. (2018) 34:953-60. doi: 10.1080/02656736.2018. 1423709

29. Eisenhauer EA, Therasse P, Bogaerts J, Schwartz LH, Sargent D, Ford R, et al. New response evaluation criteria in solid tumours: revised RECIST guideline (version 1.1). Eur J Cancer. (2009) 45:228-47. doi: 10.1016/j.ejca.2008. 10.026

30. Andocs G, Rehman MU, Zhao QL, Tabuchi Y, Kanamori M, Kondo T. Comparison of biological effects of modulated electro-hyperthermia and conventional heat treatment in human lymphoma U937 cells. Cell Death Discov. (2016) 2:16039. doi: 10.1038/cddiscovery.2016.39

31. Antonia SJ, Villegas A, Daniel D, Vicente D, Murakami S, Hui R, et al. Durvalumab after Chemoradiotherapy in stage III non-small-cell lung cancer. N Engl J Med. (2017) 377:1919-29. doi: 10.1056/NEJMoa1709937

32. Skrzypski M, Jassem J. Consolidation systemic treatment after radiochemotherapy for unresectable stage III non-small cell lung cancer. Cancer Treat Rev. (2018) 66:114-21. doi: 10.1016/j.ctrv.2018.04.001

33. Gauci ML, Lanoy E, Champiat S, Caramella C, Ammari S, Aspeslagh S, et al. Long-term survival in patients responding to anti-PD-1/PD-L1 therapy and disease outcome upon treatment discontinuation. Clin Cancer Res. (2019) 25:946-56. doi: 10.1158/1078-0432.CCR-18-0793

34. Storm FK, Harrison WH, Elliott RS, Morton DL. Normal tissue and solid tumor effects of hyperthermia in animal models and clinical trials. Cancer Res. (1979) 39:2245-51.

35. June $\mathrm{CH}$, Warshauer JT, Bluestone JA. Is autoimmunity the Achilles' heel of cancer immunotherapy? Nat Med. (2017) 23:540-7. doi: 10.1038/ nm. 4321

36. Downey SG, Klapper JA, Smith FO, Yang JC, Sherry RM, Royal RE, et al. Prognostic factors related to clinical response in patients with metastatic melanoma treated by CTL-associated antigen-4 blockade. Clin Cancer Res. (2007) 13:6681-8. doi: 10.1158/1078-0432.CCR-07-0187

37. Lisberg A, Tucker DA, Goldman JW, Wolf B, Carroll J, Hardy A, et al. Treatment-related adverse events predict improved clinical outcome in NSCLC patients on KEYNOTE-001 at a single center. Cancer Immunol Res. (2018) 6:288-94. doi: 10.1158/2326-6066.CIR-17-0063

Conflict of Interest: The authors declare that the research was conducted in the absence of any commercial or financial relationships that could be construed as a potential conflict of interest.

Copyright $\odot 2020$ Chi, Mehta, Yang, Lai, Lin, Ko, Wang, Liao and Chi. This is an open-access article distributed under the terms of the Creative Commons Attribution License (CC BY). The use, distribution or reproduction in other forums is permitted, provided the original author(s) and the copyright owner(s) are credited and that the original publication in this journal is cited, in accordance with accepted academic practice. No use, distribution or reproduction is permitted which does not comply with these terms. 